Agriculture

Canada

Research Direction générale

Branch de la recherche

Contribution 1983-11F

\title{
Conduite des vergers
}

à fortes densités

au Québec

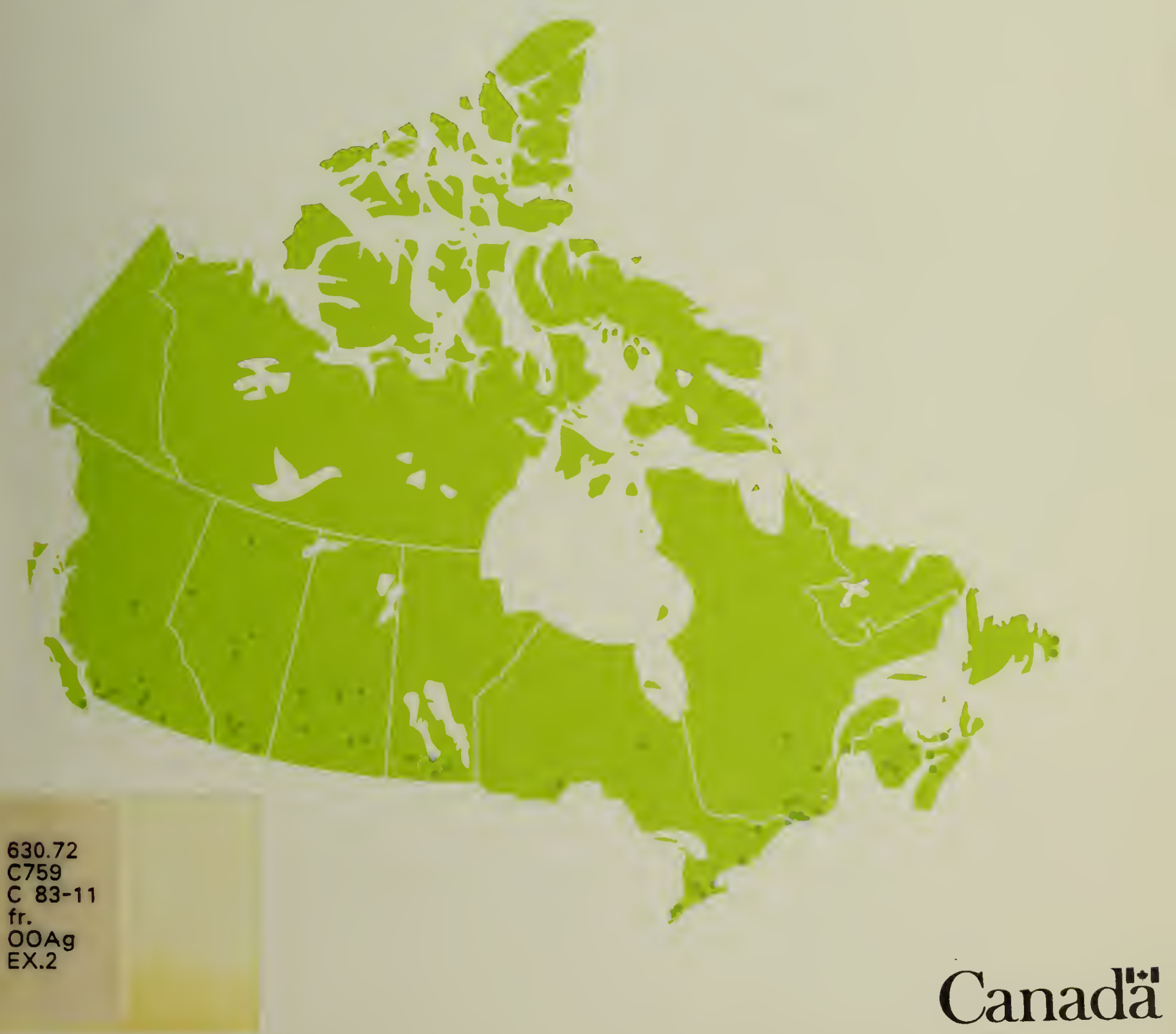




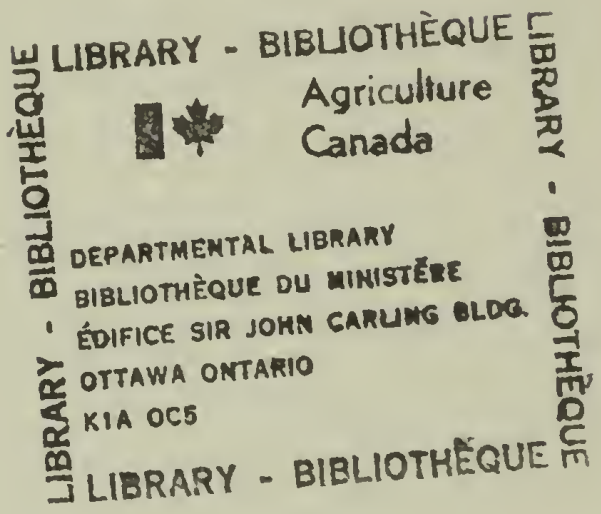




\section{Conduite des vergers à fortes densités au Québec}

RAYMOND L. GRANGER

Station de recherche

Saint-Jean-sur-Richelieu (Québec)

Bulletin technique $n^{\circ} 17$ revisé

Direction générale de la recherche Agriculture Canada

1983 
On peut oblenir de's excmplaires de ce butletin à la Station de recherche

Direction générale de la recherche

Agriculture Canada

C.P. 457

Saint-Jean-sur-Richelieu (Qućbec)

J3B 678

Production du Service aux programmes de recherche

(c) Ministre des Approvisionnements et Services Canada 1983

Also available in English under the title

Management of high-density orchards in Quebec 
Québec's fruit growing industry is now experiencing rapid and important changes. Standard size fruit trees are being replaced by dwarf and semidwarf ones. Single row systems are prevailing at the moment. The most common training systems are the "slender bell" and the "oblique palmette". Concerning apples, the 'Malling 9', 'Malling 26', 'Ottawa 3 ' and 'Malling 7' roocstocks are in prominent use. Of these, 'Ottawa 3 ' showed a superior performance. Given present economic conditions, it seems that eight hectares of dwarf apple trees planted in a high density system can viably support one Québec grower.

'Bartlett' seedlings are still the most popular rootstock for pears in this province while native plum seedlings, and the clones 'Myrobolan' and 'Brompton' are the usual plum rootstocks. Trials with other plum and pear rootstocks have been very limited. Sour and sweet cherry cultivars are mainly grafted on 'Mazzard' and 'Mahaleb' seedlings or on 'Mazzard F12' clones.

A series of questions and answers regarding some unusual aspects of the regional fruit tree culture is included.

Contribution No J 850, Research Station, Research Branch, Agriculture Canada, Saint-Jean-sur-Richelieu, Québec.

Paper presented at a colloquium on horticulture held at the International Floralies, Montréal, Québec, August 20, 1980. 

Résumé

L'arboriculture fruitière au Québec subit présentement des charigements rapides et radicaux. Les pommeraies constituées d'arbres de taille conventionnelle se transforment en plantation à fortes densités ne comportant que des arbres nains ou semi-nains. Les rangs simples y prédominent. Les nouvelles formes les plus en vogue sont la "cloche étroite" et la "palmette oblique". Chez le pommier, les porte-greffes les plus utilisés sont 1a 'Mailing 9', le 'Malling 26', 1' 'Ottawa 3' et 'Malling 7 '. Les résultats des recherches initiées à la Station de Recherches de Saint-Jean tendent à démontrer que 1' 'Ottawa 3' est supérieur. Dans la conjoncture économique actuelle, huit hectares de pommiers nains plantés à forte densité pourraient procurer à 1'arboriculteur fruitier du Québec un revenu convenable.

Pour le poirier, on y utilise encore le franc de 'Bartlett' comme portegreffe. Par ailleurs, les francs du prunier indigène, le 'Myrobolan' et le 'Brompton' sont les porte-greffes du prunier les plus employés. Les essais effectués avec d'autres porte-greffes de poirier et de prunier sont très limités. Les cultivars de griottiers et de cerisiers à fruits doux sont surtout greffés sur les francs de 'Mazzard', de 'Mahaleb' et du clone 'Mazzard F12'.

Une série de questions et réponses concernant les particularités de la pomiculture au Québec apparaît en annexe.

Contribution no J. 850 de la Station de Recherches, Direction de la Recherche, Agriculture Canada, Saint-Jean-sur-Richelieu, Québec.

Communication présentée le 20 août 1980, au Colloque International sur 1 'Horticulture patroné par les Floralies de Montréal, Québec. 
Au cours des dernières décennies, les arboriculteurs fruitiers de la plupart des pays producteurs de fruits à pépins et à noyaux ont tendance à cultiver des arbres fruitiers adaptés à leur région, petits, précoces et aptes à donner de fortes récoltes de fruits de qualité. On s'est rendu compte que la rentabilité d'une exploitation dépend d'une densité optimale d'arbres fruitiers sur une superficie donnée associée à une bonne conduite du verger.

Les arboriculteurs fruitiers du Québec sont, eux aussi, de plus en plus conscients que la culture des arbres fruitiers nains et semi-nains soumis à une régie adéquate est leur seule planche de salut. Les vergers de pommiers, en particulier ceux comportant de 90 à 180 arbres à 1 'hectare, font place à de nouvelles plantations renfermant de 600 à 3500 arbres à l'hectare. Quoique moins marquées, les mêmes tendances se manifestent quant aux poiriers, pruniers et cerisiers.

\section{Les formes}

Présentement, les formes les plus populaires au Québec sont celles de la "cloche étroite" et de la "palmette oblique" (Fig. 1 et Pl. I, figs h et $j)$. Les arbres soumis à ces formes sont surtout palissés ou tuteurés et presque toujours plantés en rangs simples. On y rencontre aussi des arbres de "plein vent" dépourvus de support.

Des essais locaux tendent à démontrer que, au point de vue rendement et rentabilité chez le pommier, des quatre formes suivantes, arbre de "plein vent", "palmette de Van Roechoudt", "palmette oblique" et "cloche étroite", les deux plus prometteuses sont la "cloche étroite" et la "pa]mette oblinue"k (Pl. I, figs h, i, j et k). La "cloche étroite" s'apparente beaucoup au "slender spindle" décrit dans ce rapport par le docteur S.J. Wertheim. Cette forme s'obtient soit en laissant la tige

* Toutes ces formes sont décrites dans le bulletin J-642 de la Station de recherches de Saint-Jean. 
Figure 1 Principales formes de pommier adoptées au Québec:

A cloche étroite ou slender spindle

B palmette oblique

A

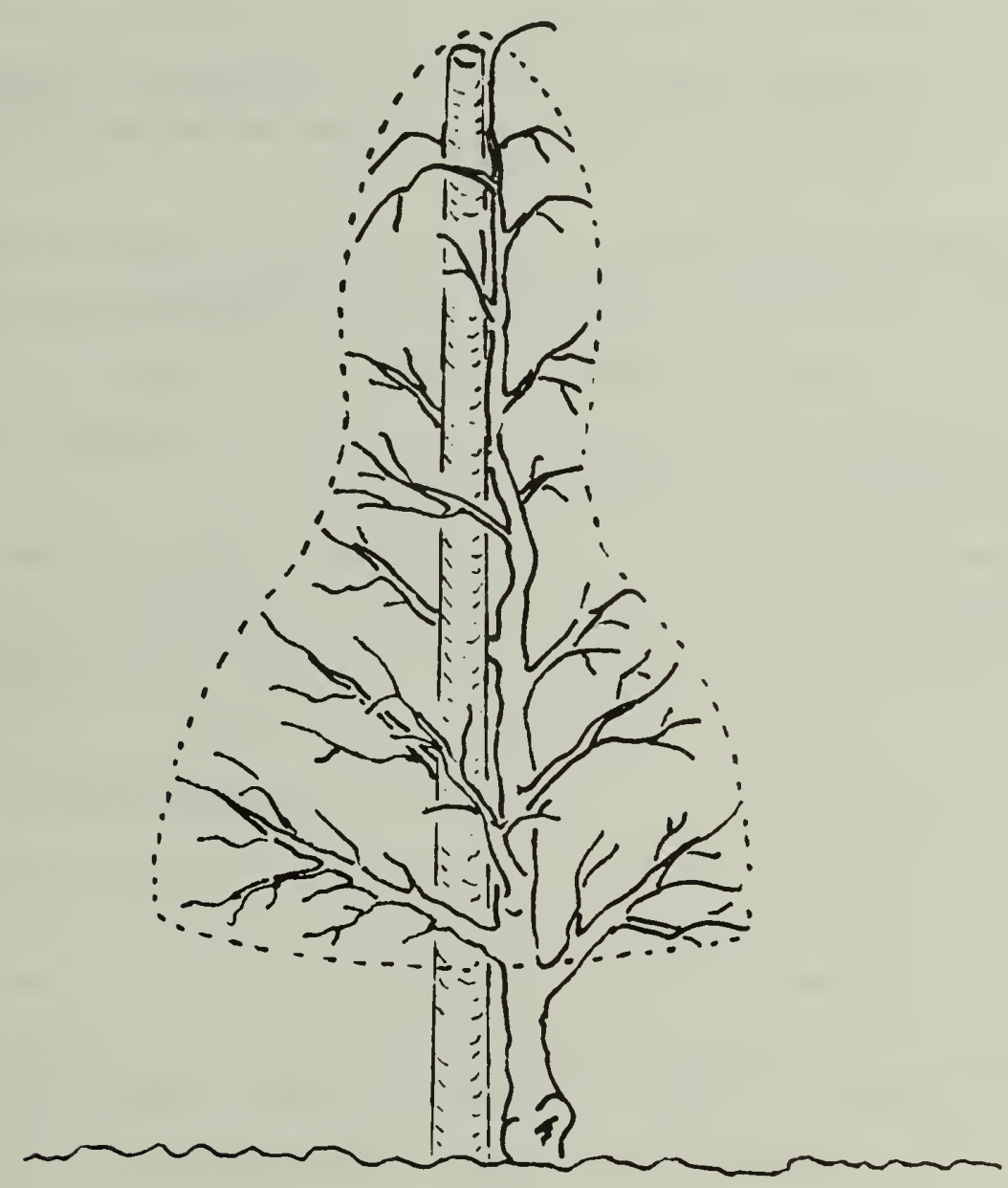

B

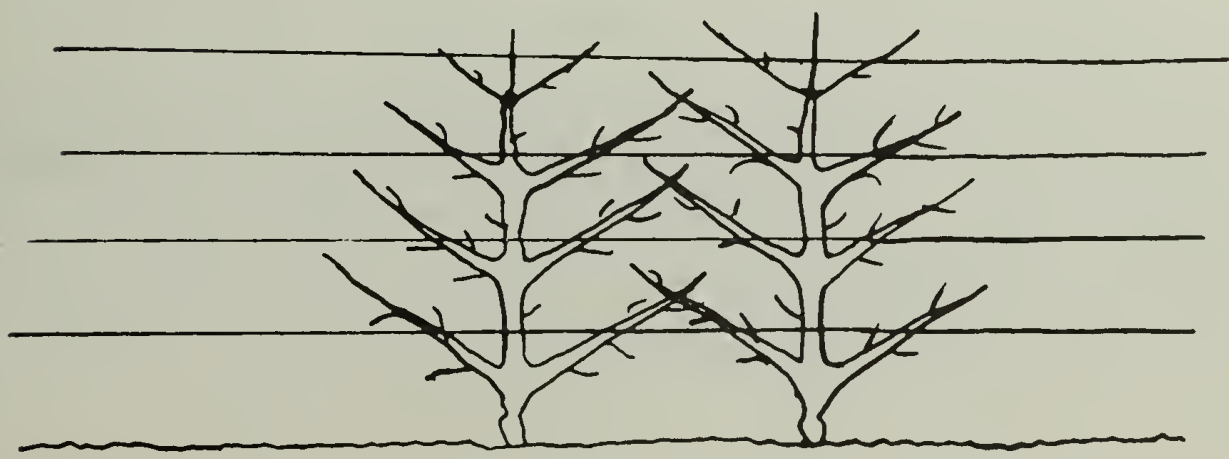


centrale intacte après la deuxième feuillaison, soit en la rabattant à chaque année, selon que 1 'on utilise la forme de "l'axe vertical" français ou celle du "slender spindle" hollandais. Dans le premier cas, il est nécessaire d'assujettir la flèche à un tuteur de bonne dimension ou à un fil de soutien élevé sans quoi la récolte aura tôt fait de la faire ployer jusqu'à ce qu'elle se brise (Pl. II, fig. g). A la sous-station expérimentale de Frelighsburg, la "cloche étroite" a favorisé une mise à fruits plus abondante et plus précoce que les autres formes (Tableau I).

TABLEAU 1 Rendements moyens ( $\mathrm{kg} / \mathrm{ha}$ ) en fonction du système de formation et de plantation de pommiers nains et semi-nains à leur $4 \mathrm{e}$ feuillaison, Frelighsburg 1979

\begin{tabular}{|c|c|c|c|c|}
\hline \multirow[t]{2}{*}{ Système de formation } & \multicolumn{3}{|c|}{ Densités** en pommiers/ha } & \multirow[t]{2}{*}{ Moyenne } \\
\hline & 2960 & 1480 & 740 & \\
\hline \multirow{5}{*}{$\begin{array}{l}\text { Cloche étroite } \\
\text { Palmette oblique } \\
\text { Plein vent } \\
\text { Palmette de Van } \\
\text { Roechoudt }\end{array}$} & $9220 \mathrm{a}$ & 5344 a & & 7282 a \\
\hline & $7320 \mathrm{~b}$ & $3870 \mathrm{c}$ & & $5595 \mathrm{~b}$ \\
\hline & & 5340 a & 3521 a & $4431 \mathrm{c}$ \\
\hline & & $3943 \mathrm{~b}$ & $1806 \mathrm{~b}$ & $2875 \mathrm{~d}$ \\
\hline & & & & \\
\hline
\end{tabular}

Suite à nos essais en cours, il semble indiqué de devoir utiliser un tuteur ou un point d'attache pour soutenir les arbres nains et parfois même les semi-nains qui, dans nos conditions climatiques, ont une forte tendance à pencher vers le sol lorsque celui-ci s'est ramolli lors de la fonte de la neige au printemps (P1. II, fig. f). Le même phénomène peut aussi se produire au cours de l'été, à cause du vent, de la pluie ou d'un poids de récolte mal réparti autour de $1^{\prime}$ axe central. Voilà pourquoi,

** Dans ce texte la densité sera qualifiée de forte (2 960 pommiers/ha), d'intermédiaire (1 480 pommiers/ha) ou de faible (740 pommiers/ha).

a Différence non significative au seuil de $5 \%$ entre les données suivies d'une même lettre. 
tel que démontré au tableau 1, les pommiers de "plein vent" accusent une diminution de rendement lorsqu' on les compare aux pommiers en "cloche étroite" et à ceux formés en "palmette oblique". Comme les pommiers formés selon la "palmette de Van Roechoudt" ont dû subir de forts émondages lors de leur $2^{e}$ et $3^{e}$ feuillaison, on peut plus difficilement les comparer aux autres pommiers au point de vue rendement.

Puisqu'au Québec le poids de la neige et du verglas tend à casser les branches inférieures des arbres nains ou semi-nains, il importe très souvent d'attacher les latérales de ces arbres fruitiers à un tuteur central ou à une palissade (P1. II, fig. e).

En ce qui concerne les poiriers, pruniers et cerisiers, la forme la plus répandue est celle de 1'arbre de "plein vent" ou de 1'arbre en "cloche" habituellement sans tuteur. Les formes palissées utilisées en ColombieBritannique chez le poirier 'Anjou' n'existent pas encore au Québec. De même, la formation de latérales fortes se prêtant à la cueillette mécanisée du griottier (cerisier à fruits amers) ne se pratique pas encore au Québec. Bien que certains arboriculteurs fruitiers aient formé leur pruniers en "gobelet", les formes les plus répandues et les plus pratiques chez le prunier au Québec demeurent celles de l'arbre de "plein vent" et de l'arbre en "cloche".

Les porte-greffes

Dans la conjoncture actuelle il devient impératif d'abaisser les coûts de production des fruits. En ce qui a trait à ceux des pommes, 1e docteur Ronald Tukey (1971) mentionne que la cueillette, la taille et la répression des ravageurs comptent pour près de $40 \%$ du total de ces coûts. Suite à d'autres études économiques effectuées dans l'état de New York (Snyder, 1968), il fut démontré que l'utilisation de pommiers nains et semi-nains contribue à diminuer considérablement les coûts de production. La même observation faite au Québec (Garon et Ouellet, 1978) nous incita à n'y recommander que les porte-greffes qui confèrent à $1^{\prime}$ arbre une 
taille d'arbre nain et de semi-nain et qui sont adaptés à nos conditions climatiques. Tels sont le 'Malling 26', '1'Ottawa 3', le 'Malling 7' et le 'Malling 9', ce dernier, toutefois, ne devant être employé qu'avec réserve.

Le 'Malling 9'

Le 'Malling 9' est un porte-greffe nanisant mis à 1'essai à la Station de Recherches d'Ottawa de 1938 à 1970. I1 s'y est montré sensible au froid (Davis et al., 1948). En Russie, on le considère également comme très peu rustique (Margolin, 1951 et Smirnov, 1960). Au Québec, le 'Malling 9'. cultivé en terrain engazonné près du Collège Macdonald, a résisté à nos hivers depuis au moins 22 ans (Taper, 1979). Cependant, après leur première feuillaison, des pommiers mal aoûtés des cultivars 'McIntosh' et 'Spartan' sur 'Malling 9', qui poussaient en terrain désherbé à la sousstation de Frelighsburg, ont souffert considérablement du froid au cours de 1'hiver 1975-1976 (Rousselle et al., 1976).

Dans nos parcelles d'expérimentation de Frelighsburg, le 'Malling 9 'en bas âge fut le moins productif des porte-greffes de type nain (Tableau 2).

Ce porte-greffe semble se bien comporter avec les cultivars les mieux adaptés aux plantations denses comme 'l'Empire'*, le 'Golden Delicious'* et le 'Red Haralson' $* *$ (P1. I, e et f).

A Vineland en Ontario, des pommiers 'McIntosh', 'Cortland', 'Red Delicious', tous sur 'Malling 9', ont rapporté en moyenne deux boisseaux par pommier de la 5 e à la 10e feuillaison. A ce même endroit, des pommiers 'McIntosh', 'Spy' et 'Red Delicious' sur 'Malling 9', considérés comme relativement âgés, ont donné, entre 20 et 25 ans, une moyenne de $54 \mathrm{~kg}$ ( 3 boisseaux) de pommes par arbre (Hutchinson, 1964). Certains de ces arbres ont fourni jusqu'à $72 \mathrm{~kg}$ ( 4 boisseaux). A Ottawa, le rendement annuel des pommiers 'McIntosh' sur 'Malling 9' a été de 16 à $27 \mathrm{~kg}$

* cultivar sensible au froid, ne se cultive qu'en verger protégé du froid *** donne des fruits de mauvaise qualité si cultivé dans une zone à climat froid 
par arbre lorsque ceux-ci ont eu de 12 à 25 ans. Le 'Malling 9' exige un sol passablement lourd et bien pourvu d'eau mais bien aéré et bien égoutté (Granger et al., 1974).

TABLEAU 2 Rendements moyens ( $\mathrm{kg} / \mathrm{ha}$ ) des cultivars 'McIntosh' et 'Spartan' en fonction des porte-greffes et des densités de plantation de pommiers nains et semi-nains à leur $4 \mathrm{e}$ feuillaison, Frelighsburg 1979

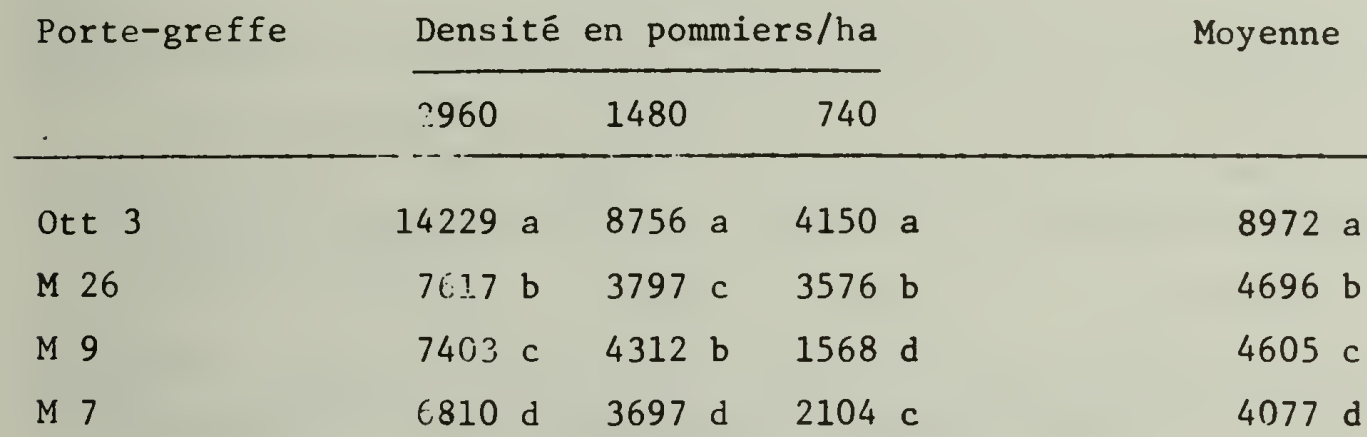

a Différence non significative au seuil de $5 \%$ entre les données suivies d'une même lettre.

D'après les résultats obtenus dans nos parcelles de Frelighsburg avec les cultivars 'Spartan' et 'McIntosh' sur 'Malling 9', il semble que la densité optimale pour ce porte-greffe se situe aux environs de 2960 arbres à l'hectare lorsque les arbres sont formés en "cloche étroite". Dans le cas des autres formes sur 'Malling 9', c'est-à-dire chez les arbres de "plein vent", de la "palmette oblique" et de la "palmette de Van Roechoudt", e'est également à forte densité qu'ils atteignent leur rendement optimal par arbre. Ces densités sont respectivement de 2960 et de 1480 arbres à 1 'hectare pour les formes "cloche étroite" et "palmette oblique" tandis qu'elles sont de 1480 et de 740 arbres à l'hectare pour les formes "palmettes de Van Roechoudt" et d'arbres de "plein vent".

Depuis quelques années, certains de nos pomiculteurs trouvent que les nouveaux 'Malling 9' exempts de viroses sont trop vigoureux et pas aussi productifs que les anciens qui étaient porteurs de maladies virales. 
On note également que, dans nos conditions climatiques très humides, le 'Malling $9^{\prime}$ a tendance à former des galles radiculaires aériennes appelées en anglais burr knots; ces galles entravent la bonne circulation de la sève et rendent l'arbre plus vulnérable au froid et à certaines maladies (P1. II, fig. d).

Le 'Malling 26 '

Créé en 1929 à la Station de Recherches de East Malling en Angleterre par suite du croisement 'Malling $16^{\prime} \mathrm{x}$ 'Malling 9', le 'Malling 26' fut distribué pendant un certain temps sous le numéro 3426. Ce porte-greffe est actuellement $I^{\prime}$ un des plus vendus au Canada. Il a fait l'objet d'essais à la Station de Recherches d'Ottawa et continue de le faire aux Stations de Recherches de Saint-Jean au Québec, de Smithfield, Vineland, Harrow, Simcoe et Guelph en Ontario et de Kentville en Nouvelle-Ecosse (Granger et al., 1974).

A Vineland, Ontario, le 'Malling 26' a permis d'obtenir une moyenne de deux boisseaux de la 5 e à la 10e feuillaison. A Frelighsburg, il a, avec les cultivars 'Spartan' et 'McIntosh', donné en bas âge un rendement supérieur à celui du 'Malling 9' et s'y est classé deuxième (Tableau 2). Il faut admettre que 1 e 'Malling $26^{\prime}$ est plus compatibles avec le cultivar 'McIntosh' que ne 1 'est le 'Malling 9' (Tableau 3).

Lorsque cultivé dans un sol qui lui convient. c'est-à-dire en terre franche graveleuse bien égouttée et à fort pouvoir de rétention d'eau, le 'Malling $26^{\prime}$ occasionne une pousse végétative plus abondante que celle apportée par le 'Malling $9^{\prime}$. Dans nos parcelles expérimentales, le 'Malling $26^{\prime}$ a produit une croissance végétative plus forte que le 'Malling 9', ce qui a nécessité un émondage plus élaboré (Tableau 4).

Parmi les porte-greffes du type nain le 'M-26' est celui qui a produit la plus forte proportion de gros fruits tel que révélé par le poids moyen des pommes (Tableau 5). 
TABLEAU 3 Rendements moyens ( $\mathrm{kg} / \mathrm{ha}$ ) de pommiers nains et semi-nains à leur $4^{\mathrm{e}}$ feuillaison, Frelighsburg 1979

\begin{tabular}{lll}
\hline Porte-greffe & \multicolumn{2}{c}{ Cultivar } \\
\cline { 2 - 3 } & Spartan & McIntosh \\
\hline Ott 3 & $8972 \mathrm{a}$ & $8691 \mathrm{a}$ \\
M 9 & $6564 \mathrm{~b}$ & $2552 \mathrm{~d}$ \\
M 26 & $5206 \mathrm{c}$ & $4186 \mathrm{c}$ \\
M 7 & $3298 \mathrm{~d}$ & $4855 \mathrm{~b}$ \\
\hline
\end{tabular}

a Différence non significative au seuil de $5 \%$ entre les données suivics d'une même lettre.

TABLEAU 4 Diamètre moyen $(\mathrm{cm})$ du tronc de pommiers nains et semi-nains à leur $4^{\mathrm{e}}$ feuillaison, Frelighsburg 1979

\begin{tabular}{lllll}
\hline \multirow{2}{*}{ Porte-greffe } & \multicolumn{2}{l}{ Densité en pommiers/ha } & Moyenne \\
\cline { 2 - 5 } & 2960 & 1480 & 740 & \\
\hline M 7 & $4.00 \mathrm{a}$ & $4.32 \mathrm{a}$ & $4.49 \mathrm{a}$ & $4.27 \mathrm{a}$ \\
M 26 & $3.85 \mathrm{ab}$ & $3.84 \mathrm{~b}$ & $4.44 \mathrm{a}$ & $4.04 \mathrm{ab}$ \\
Ntt 3 & $3.65 \mathrm{~b}$ & $3.87 \mathrm{~b}$ & $3.94 \mathrm{~b}$ & $3.82 \mathrm{~b}$ \\
M 9 & $2.83 \mathrm{c}$ & $2.99 \mathrm{c}$ & $3.01 \mathrm{c}$ & $2.94 \mathrm{c}$ \\
\hline
\end{tabular}

a Différence non significative au seuil de $5 \%$ entre les données suivies d'une même lettre.

Sous notre climat, le 'Malling $26^{\prime}$ s'est avéré beaucoup plus rustique que le 'Malling 9' (Rousselle et al., 1976). Introduit il y a dix ans dans notre région, ce porte-greffe n'a pas été encore affecté par le froid. Selon les rapports de la Station de Recherches d'Ottawa, le M-26 serait plus sensible au froid que le 'Malus robusta 5' mais, au Minnesota, ces deux porte-greffes auraient été aussi rustiques 1 'un que 1 'autre (Watkins, 1968; Granger et al., 1974). 
TABLEAU 5 Poids moyen (g/fruit) des fruits de pommiers nains et seminains à leur 4e feuillaison, Frelighsburg 1979

\begin{tabular}{lllll}
\hline Porte-greffe & \multicolumn{3}{c}{ Densité en pommiers/ha } & Moyenne \\
\cline { 2 - 4 } & 2960 & 1480 & 740 & $161 \mathrm{a}$ \\
\hline M 26 & $154 \mathrm{c}$ & $166 \mathrm{a}$ & $160 \mathrm{~b}$ & $159 \mathrm{~b}$ \\
M 9 & $159 \mathrm{a}$ & $160 \mathrm{~b}$ & $161 \mathrm{a}$ & $151 \mathrm{c}$ \\
Ott 3 & $156 \mathrm{~b}$ & $155 \mathrm{c}$ & $140 \mathrm{c}$ & $150 \mathrm{~d} *$ \\
\hline
\end{tabular}

a Différence non significative au seuil de $5 \%$ entre les données suivies d'une même lettre.

* une pomme de 150 grammes est considérée comme étant d'un calibre acceptable au point de vue commercial

Au Québec, le 'Malling 26' s'est montré sensible à la brûlure bactérienne (Emvin ia amylovora Burr. Winsl. et al.), quoique, après greffage, cette maladie affecte rarement le porte-greffe lui-même. La plus importante anomalie observée sur le 'Malling 26' au Québec réside dans sa forte tendance à développer des galles radiculaires aériennes ou burr knots (P1. II, fig. d). Pour obvier à cette tendance, on peut bagigeonner la partie aérienne du 'Malling 26' avec une solution à $1 \%$ d'acide naphtalène acétique (ANA ou NAA). Cette solution peut se mélanger à un peu de peinture blanche de type "Latex". Il est important de traiter les galles dès qu'elles apparaissent sans quoi elles formeront de larges plaques qui entraveront la circulation de la sève. Il est aussi tout à fait contre-indiqué d'utiliser autour des troncs des protecteurs mal ajourés car ils contribuent à maintenir la couronne et la base aérienne de l'arbre dans une atmosphère d'humidité constante, ce qui favorise la formation de ces galles. Il importe aussi d'éliminer les hautes herbes près des pommiers car elles maintiennent également un fort pourcentage d'humidité au pied des arbres. S'il y a lieu d'utiliser les protecteurs contre les campagnols des champs, on devra donc employer de préférence ceux qui sont faits en treillis métalliques. Notons enfin que le 'Malling 26' se comporte très mal dans un sol trop léger et trop sec. 
En pareil cas, il est à conseiller d'avoir recours à l'irrigation.

L'Ottawa 3'

Créé à la Station de Recherches d'Ottawa, ce nouveau porte-greffe est le résultat du croisement 'Robin' par 'Malling 9' (1956). De 1967 à 1973, il fut mis à l'essai à Ottawa ainsi qu'à Smithfield en Ontario. Multiplié et évalué à la sous-station de Frelighsburg depuis ce temps, il a été distribué à plusieurs pépiniéristes du Canada afin de répondre aux demandes les plus pressantes des pomiculteurs.

Les cultivars 'McIntosh' et 'Quinte' greffés sur 'Ottawa 3' depuis 1967 sont demeurés des pommiers nains, c'est-à-dire légèrement plus petits que sur 'Malling 26' mais un peu plus gros que sur 'Malling 9'. A maturité, un pommier McIntosh' sur 'Ottawa $3^{\prime}$ peut être maintenu à une hauteur légèrement inférieure à trois mètres; les fruits sont par le fait même très faciles à cueillir (Granger et al., 1974).

L''Ottawa $3^{\prime}$ s'est avéré très rustique, se comparant favorablement au Malus robusta 5. Dans 1'est du pays, la survie des pommiers, lors de conditions climatiques défavorables, a quelquefois été assurée par la rusticité de leurs porte-greffes. Ces conditions peuvent prévaloir encore. Il est donc important d'utiliser autant que possible des portegreffes rustiques.

L'Ottawa 3' a favorisé de très bonnes récoltes compte tenu de la faible taille qu'il confère au pommier. Ainsi, des arbres de deux ans de 'Quinte et 'McIntosh' sur 'Ottawa 3', plantés en 1967 à Ottawa, commencèrent à rapporter en 1970 ( $4^{\mathrm{e}}$ feuillaison), fournissant une récolte moyenne de $5,45 \mathrm{~kg}$ par arbre pour atteindre $18 \mathrm{~kg}$ en 1972 et 1973 (6 $6^{\mathrm{e}}$ et 7 feuillaison). Les rendements des pommiers greffés sur 'Ottawa 3 ' se sont alors avérés supérieurs à ceux provenant de pommiers sur 'Malling 26'. De plus, 'Ottawa 3' a produit des pommes 'McIntosh' et 'Quinte' un peu plus hâtivement que le 'Malling 26', mais la qualité des fruits 
récoltés sur ces deux porte-greffes a été excellente. A la sous-station de Frelighsburg, les résultats obtenus avec 'Ottawa 3 ' ont été semblables à ceux cités ci-dessus. Cependant, avec les formes qui lui sont les plus appropriées, 1''Ottawa $3^{\prime}$ a donné lieu à de meilleurs rendements à Frelighsburg qu'à Ottawa. En effet le tableau 2 nous indique qu'avec les cultivars 'McIntosh' et 'Spartan' et avec des arbres formés selon les quatre méthodes mentionnées au début $1^{\prime \prime}$ 'Ottawa $3^{\prime} \mathrm{s}^{\prime}$ avère de loin le porte-greffe qui permet d'obtenir de forts rendements hâtifs de fruits de qualité.

La forme qui semble convenir le mieux à 1'"Ottawa 3' est la "cloche étroite" (Tableaux 6 et 7).

TABLEAU 6 Rendements moyens ( $\mathrm{kg} / \mathrm{ha}$ ) de pommiers 'Spartan' nains et seminains formés en "cloche étroite" (1) en "palmette obliqque" (2) à leur $4^{\mathrm{e}}$ feuillaison, Frelighsburg 1979

Porte-greffe

Densité pommiers/ha

Moyenne

\begin{tabular}{|c|c|c|c|c|c|c|}
\hline & & & \\
\hline & 2960 & & 1480 & & & \\
\hline & (1) & (2) & (1) & (2) & (1) & (2) \\
\hline Ott 3 & 15170 a & 13287 a & 11350 a & $76: 25 \mathrm{a}$ & 13260 a & 10458 \\
\hline M 9 & $15537 \mathrm{a}$ & $9048 \mathrm{~b}$ & $7448 \mathrm{~b}$ & $3643 c$ & $11493 \mathrm{~b}$ & 6346 \\
\hline M 26 & 8962 b & $6029 c$ & $5347 c$ & $5260 \mathrm{~b}$ & $7155 \mathrm{c}$ & 5645 \\
\hline M 7 & $6621 \mathrm{c}$ & $7790 \mathrm{~d}$ & $3825 \mathrm{~d}$ & $1370 \mathrm{~d}$ & $5223 \mathrm{~d}$ & 4580 \\
\hline
\end{tabular}

a Différence non significative au seuil de $5 \%$ entre les données suivies d'une même lettre.

Avec cette forme, cependant, lorsque planté à forte densité, le cultivar 'Spartan' sur 'Ottawa $3^{\prime}$ ne s'est pas montré significativement supérieur au 'Malling 9'. A cette densité les fruits du 'Spartan' sur 'Ottawa 3' ont eu tendance à être petits, ce qui a diminué le poids de la récolte. La forme qui convient le moins bien au cultivar 'Spartan' sur 'Ottawa $3^{\prime}$ est celle de 1 'arbre de "plein vent" non tuteuré (Tableaux 6 et 7 ). 
TABLEAU 7 Rendements moyens ( $\mathrm{kg} / \mathrm{ha}$ ) de pommiers 'Spartan' nains et seminains formés en arbres de "plein vent" ( 1 ) et en "palmette de Van Roechoudt" (2) à leur 4e feuillaison, Frelighsburg 1979

\begin{tabular}{|c|c|c|c|c|c|c|}
\hline \multirow[t]{2}{*}{ Porte-greffe } & \multicolumn{4}{|c|}{ Densité pommiers/ha } & \multicolumn{2}{|c|}{ Moyenne } \\
\hline & 1480 & & 740 & & & \\
\hline & (1) & (2) & (1) & (2) & (I) & (2) \\
\hline $0 t t 3$ & 8242 a & 7807 a & 3280 a & 5019 a & 5761 a & $6413 a$ \\
\hline M 9 & $7886 \mathrm{~b}$ & $3520 \mathrm{c}$ & 3125 a & $2309 \mathrm{~b}$ & 5505 a & $2915 \mathrm{~b}$ \\
\hline M 26 & $4141 \mathrm{c}$ & $4590 \mathrm{~b}$ & $5820 \mathrm{~b}$ & $1498 \mathrm{c}$ & $4980 \mathrm{~b}$ & $3044 \mathrm{~b}$ \\
\hline$M 7$ & $3224 d$ & $1361 \mathrm{~d}$ & $1580 \mathrm{c}$ & $598 \mathrm{~d}$ & $2411 c$ & 978 \\
\hline
\end{tabular}

a Différence non significative au seuil de $5 \%$ entre les données suivies d'une même lettre.

Cela nous indique qu'un pommier avec porte-greffe 'Ottawa $3^{\prime}$ a besoin d'un tuteur ou d'un support quelconque. Les mensurations des arbres de nos parcelles de Frelighsburg confirment les observations antécédentes, à savoir que 1 'Ottawa $3^{\prime}$ confère au pommier un degré de vigueur qui le situe entre le 'Malling 9' et le 'Malling 26' (Tableau 4). Il est bon de noter qu'une trop forte densité (quelle que soit la forme adoptée) est de nature à diminuer la vigueur des pommiers sur 'Ottawa 3' (Tableau 4). En général, des densités intermédiaires (toutes les formes combinées) permettent à 1''Ottawa 3 ' d'obtenir son équilibre physiologique optimal. Là où il atteint sa performance maximale, c'est-à-dire là où chaque arbre rapporte le plus individuellement c'est avec la forme de "cloche étroite" à une densité intermédiaire de 1480 arbres à 1 'hectare. Par contre, les plus hauts rendements à l'hectare furent obtenus lorsque des pommiers 'Spartan'/'Ottawa 3' furent plantés à forte densité même si, dans ces conditions, le rendement moyen par arbre a été plus faible (Tableaux 6 et 7). L' 'Ottawa 3' semble être un aussi bon porte-greffe pour le cultivar 'McIntosh' que pour le cultivar 'Spartan', alors que le 'Malling 26' de même que le 'Malling 9' s'avèrent de moins bon portegreffes pour le 'McIntosh' que pour le 'Spartan' (Tableau 3). Toutefois, 
lorsque non irrigués et non soumis à l'éclaircissage chimique les pommiers 'McIntosh'/'Ottawa 3' et 'Spartan'/'Ottawa 3' ont tendance à donner des pommes de calibre plutôt faible (Tableau 5).

Employé comme tige intermédiaire de 8 à 10 pouces sur le porte-greffe 'Malus robusta 5', 1''Ottawa 3 ' a donné d'excellents rendements hâtifs à Ottawa et à Frelighsburg. Les cultivars 'McIntosh' et 'Quinte' greffés de la sorte ont donné un rendement par arbre approximativement deux fois supérieur à celui fourni par ces mêmes cultivars lorsque greffés directement sur racines 'Ottawa 3'. L''Ottawa 3' s'est avéré supérieur au 'Malling 26' lorsqu'employé comme tige intermédiaire sur 'Malus robusta $5^{\prime}$.

Jusqu'à date, 1''Ottawa $3^{\prime}$ n'a pas semblé trop sensible aux maladies virales. Dans certains cas, cependant, des scions, tels que ceux du 'Jerseymac' et du 'Macspur' non indexés, lui ont transmis des viroses dont les symptômes se sont manifestés au niveau de la greffe et se sont traduits par des rayures brunes sous l'écorce. Les arbres ainsi affectés sont destinés à mourir à plus ou moins brève échéance. De plus, en pépinière, ce clone est très sensible au mildiou poudreux et au puceron lanigère.

Ces inconvénients seraient plutôt négligeables en verger, mais il vaut mieux quand même prévenir les pomiculteurs et les pépiniéristes afin que ceux-ci ne greffent sur 'Ottawa $3^{\prime}$ que des scions indexés quant aux principales viroses.

La mulplication de 1''Ottawa $3^{\prime}$ est très difficile en cépée mais relativement facile par bouturage de racines. Les boutures de racines d'Ottawa 3' gardées en serre, de février à mai, à la Station de Recherches de Saint-Jean ont repris aux taux respectifs de 600 et de 850 sur 1000 en 1972 et 1973. Lorsque multiplié par boutures de bois non aoûté, ce clone a eu un pourcentage d'enracinement variant de 62 à $65 \%$ de 1969 à 1974 . Ce pourcentage est inférieur à celui obtenu avec le 'Malus robusta 5' 
mais supérieur à celui obtenu avec le 'Malling 7'.

\section{Lee 'Ma11ing 7'}

Ce porte-greffe, connu depuis le XVIIe siècle en France et en Angleterre, a été resélectionné en 1959 par la Station de Recherches d'East Malling en Angleterre (Tukey, 1964). C'est le seul porte-greffe actuellement disponible mondialement qui confère au pommier une taille d'arbre seminain.

Le 'Malling 7', étant un des plus vieux porte-greffes utilisés en pomiculture, est considéré en Amérique du Nord comme l'un des plus sûrs car il s'est relativement bien comporté dans une multitude de conditions de sols et de climats. Au Massachusetts et au Michigan, on lui accorde encore une place de choix au sein des nouvelles plantations. On y préfère ne pas utiliser de tuteurs et donner aux arbres un minimum de soins d'entretien. Le 'Malling 7' est précisément le porte-greffe qui, dans ces conditions peut quand même se comporter de façon rentable (Lord et Costante, 1977). Avec le 'Malling 7' comme porte-greffe, des pommiers non tuteurés soumis à un émondage presque nul et croissant sans soins particuliers dans un sol mal égoutté et envahi par les mauvaises herbes, se sont relativement bien comportés depuis 1957 à Frelighsburg (Granger, 1972). A leur $9^{\mathrm{e}}$ et $10^{\mathrm{e}}$ feuillaison des pommiers, des cultivars 'Melba', 'McIntosh' et 'Bancroft' sur 'Malling 7' ont eu de faibles rendements allant de 20304 à $38394 \mathrm{~kg} / \mathrm{ha}$ (moyenne des deux années et des trois cultivars, combinés) mais les fruits en furent d'excellente qualité. A faible densité, les rendements à 1 'hectare chez des pommiers greffés sur 'Malus robusta 5' ont été légèrement supérieurs à ceux des arbres greffés sur 'Malling 7', soit 18770 contre $18246 \mathrm{~kg} / \mathrm{ha}$. Toutefois, à la densité maximale, les rendements des cultivars sur 'Malling 7 ' ont été supérieurs à ceux des cultivars sur 'Malus robusta 5', 1'augmentation étant de $1^{\prime}$ ordre de $1778 \mathrm{~kg} / \mathrm{ha}$ en faveur du 'Malling 7' pour les ge et $10 \mathrm{e}$ feuillaisons combinées. Des observations plus récentes à la sous-station de Frelighsburg nous ont révélé que, parmi les 4 porte-greffes essayés, 
le 'Malling 7' est celui qui a fourni les rendements les plus bas autant à forte qu'à faible densité, exception faite pour le 'McIntosh' qui, greffé sur 'Malling 7', donna plus de pommes que lorsque greffé sur 'Màlling 9' ou 'Malling 26' (Tableaux 2 et 3). Le 'Malling 7' semble donc mieux s'adapter à la 'McIntosh' que le 'Malling 9' ou le 'Malling 26' (Tableau 3). Nous avons constaté que la forme qui convient le mieux au 'Malling 7 ' est celle de la "cloche étroite" et que, même avec ce porte-greffe, 11 importe de supporter les arbres sans quoi les rendements diminuent (Tableaux 6 et 7 ). Enfin, le 'Malling 7 ' s'est montré plus vigoureux que les quatre autres porte-greffes et a donné les plus gros fruits (Tableaux 4 et 5 ).

Le 'Malling 7' a un système radiculaire pivotant et non pas traçant comme c'est le cas pour les trois autres porte-greffes dont il fut question. De ce fait, il peut, du moins dans certaines conditions (gel partiel de ses racines, etc.), souffrir d'une carence en potassium. De plus, 11 a une forte tendance à produire des drageons et il s'est montré très sensible à la tumeur du collet, (Agrobacterium tumefaciens) (P1. III, fig. h). Même si le recours à ce porte-greffe semble être une solution facile dans l'esprit de plusieurs pomiculteurs du Québec suite à ce qu'ils ont observé au Massachusetts et ailleurs, une mise en garde s'improse; certes le 'Malling 7' est un porte-greffe sûr pour le pomiculteur qui, avec un minimum d'efforts, veut obtenir des petites ou de moyennes récoltes. Toutefois le 'Malling 7 ' n'est pas du tout à conseiller à celui qui tient à obtenir du volume et de la qualité, c'est-à-dire à celui qui tient à rentabiliser le plus possible son exploitation. Les recherches effectuées à Vineland, à Smithfield, à Saint-Jean et à Ottawa mettent en évidence la productivité plutôt faible du 'Malling 7' (Hutchinson, 1964).

Porte-greffes de poiriers

Le porte-greffe de poiriers le plus répandu maintenant au Québec est le franc de 'Bartlett' ('Williams'). Quoique peu précoce et assez sensible à la brûlure bactérienne (Erwinia comylovora Burr. Winsl et al.), ce genre 
de porte-greffe s'est avéré plus rustique et plus vigoureux que certains cognassiers non adaptés à notre climat, surtout le 'cognassier C'. Toutefois 1e 'Pyrus ussuriensis' s'est avéré le plus rustique et le plus fiable de tous les porte-greffes de poiriers essayés au Québec.

Le 'cognassier d'Angers', que 1 'on considère comme passablement rustique en Ontario et à la station de recherches de Geneva, N.Y. (Lapins 1963), n'a subi que quelques essais au Québec. Les francs de 'Bartlett' se sont montrés compatibles avec les cultivars les plus cultivés au Québec, nommément le 'Flemish Beauty', le 'Clapp's Favorite', 'l'Anjou', 1e 'Bosc', et le 'Bartlett' (Pl. III, figs b, c, d). Ce dernier, en raison de son manque de rusticité, n'a pas été rentable au Québec.

Depuis 1972, nous avons essayé à Frelighsburg, des francs d'aubépine indigère (Crataegus L.) dans le but de naniser le poirier et d'en hâter la mise à fruits. Nous avons noté que la longévité des scions de poiriers sur aubépine était très courte. En général, une telle combinaison ne dure que cinq ou six ans. Nous avons ensuite mis à I'essai des pièces intermédiaires de 'Old Home' sur racines de Crataegus. Selon des résultats obtenus en Oregon, cette combinaison devait conférer au poirier une certaine résistance à la brûlure bactérienne (Erwinia amylovora Burr. Winsl et al.), une fructification plus précoce, ainsi qu'un nanisme mitigé. Malheureusement, le taux de reprise des arbres comportant ces doubles greffes n'a été que de $25 \%$; toutefois, les sujets qui ont repris se sont bien comportés, ont commencé à rapporter tôt et sont devenus des poiriers semi-nains. La meilleure combinaison a été celle du scion sur 'Old Home' sur franc. Suite à ces essais, certains de nos pépiniéristes ont commencé à utiliser le 'old Home' comme pièce intermédiaire sur franc. Des essais préliminaires nous révèlent que le 'Harbin' et le 'Luscious' pourraient avantageusement servir de porte-greffes de poirier au Québec. Nous n'avons pas encore essayé aucun des porte-greffes russes et allemands tels les cognassiers 'Melitopolskaya', et 'Severnaya', et les sélections de cognassiers 'Pillnitz' 非, 3 et 5 ou les clones 'Pillnitz' ('Pyrus betulifolia') reconnus comme relativement nanisants au froid. 


\section{Les porte-greffes de pminiers}

Depuis au-delà d'un demi-siècle, les porte-greffes de prunier les plus fréquemment employés au Québec ont été les francs du prunier indigène ('Pmunus comericana') et le 'Myrobolan B'. d'East Malling. Avec de tels porte-greffes, les pruniers ont été plantés à la densité d'environ 450 arbres à l'hectare. Aux sous-stations de La Pocatière et de Frelighsburg, nous avons planté des pruniers greffés sur le porte-greffe 'Brompton' au taux de 840 arbres à l'hectare. Cette expérience, initiée en 1977, comporte plus de 30 cultivars de pruniers sur ce porte-greffe; ces arbres ont commencé à produire mais il est encore trop tôt pour faire état des résultats obtenus.

Aux stations de recherches d'Ottawa, de Vineland et de Geneva, des essais ont porté sur l'action nanisante des francs du 'cerisier des sables' ('Prunus besseyi') employés comme porte-greffes (Blair, 1954; Hutchinson, 1964). Les rendements ont été trouvés acceptables, la maturité des fruits a été de 7 à 10 jours plus hâtive et leur qualité a été excellente. Cependant, il a fallu supporter les arbres avec de solides tuteurs et il semble que les scions du type 'Damson' n'ont pas été compatibles avec ces porte-greffes. A la sous-station de Frelighsburg, nous avons greffé des pruniers sur 'Prunus tomentosa' mais la reprise ne fut que de $15 \%$. Ces arbres avaient une taille environ deux fois moindre que celle des arbres standards et auraient pu être plantés à des densités excédant 1500 arbres à l'hectare.

Porte-greffes de griottiers et de cerisiers

Les porte-greffes de griottiers les plus répandus au Québec sont les francs de 'Mazzard' et de 'Mahaleb' et le clone 'Mazzard F12'. Bien que les francs de 'Mahaleb' soient légèrement nanisants pour les griottiers comme 'Montmorency', 'Northstar' et 'Meteor' ils en font malgré tout des arbres qui atteignent rarement plus que $50 \%$ ou $75 \%$ des dimensions des arbres standards. Lapins (1963) rapporte qu'une pièce intermédiaire du 
petit merisier d'Amérique ('Prunus pensylvanica') sur racine de 'Mazzard' a une forte action nanisante tout en étant compatible avec les principaux griottiers commerciaux. A la sous-station de Frelighsburg, nous avons fait plusieurs essais avec des francs de 'merisiers d'Amérique' qui ont toujours semblé compatibles avec les scions de griottiers commerciaux. Il est toutefois encore trop tôt pour faire état des résultats obtenus. Comme nous n'avons pas encore essayé le porte-greffe nanisant 'Colt' d'Angleterre, nous n'avons pas de porte-greffes qui nous permettent vraiment de planter des griottiers et des cerisiers (pour jardiniers amateurs) à forte densité au Québec.

\section{Aspects économiques}

Même si au Québec il est un peu tôt pour pouvoir nous prononcer sur la rentabilité des formes d'arbres fruitiers cultivés à telle ou telle densité, les premiers résultats obtenus dans nos parcelles expérimentales et chez des producteurs avant-gardistes sont de bon augure. D'ailleurs Fisher (1969) en Colombie-Britannique, Norton (1971) dans l'Etat de New York et Hutchinson (1976) et Miller (1976) en Ontario ont rapporté que, même en Amérique, les vergers à fortes densités étaient plus rentables que les vergers conventionnels. Il va sans dire que, selon ces mêmes chercheurs, les plantations trop denses n'ont pas été trouvées plus rentables que celles qui ne l'étaient pas assez.

Chez le pommier, le bilan de nos parcelles expérimentales à Frelighsburg nous indique que pour 1'année 1979 ( $4^{\mathrm{e}}$ feuillaison) la forme de "cloche étroite" avec arbres plantés à forte densité fut la combinaison la plus rentable (bénifice de 2042,50 \$à 1 'hectare). Elle a été suivie par la "palmette oblique" à forte densité (bénifice de 1457 \$à 1'hectare). A partir des données que nous possédons présentement, il est difficile de prédire quand nos pommiers atteindront leur seuil de rentabilité. Nous prévoyons que, au rythme où ils progressent avec la meilleure combinaison possible, soit 'McIntosh' ou 'Spartan' sur 'Ottawa 3' plantés à forte densité en forme de cloche étroite, nous pourrons vers $1 \mathrm{a} 6^{\mathrm{e}}$ feuillaison 
annuler complètement la dette initiale de 16342 \$ l'hectare qu'il en a coûté pour l'établissement et l'entretien jusqu'en 1979 de ce système. En somme, il faudra que, chez ces arbres, la production passe de $9220 \mathrm{~kg} /$ ha à environ $40000 \mathrm{~kg} / \mathrm{ha}$ en 1981. Certes les arbres de plein vent furent ceux qui nous ont coûté le moins cher à établir et à entretenir mais il semble que ce ne seront pas ceux qui vont s'avérer les plus rentables. Certains pomiculteurs qui ont des pommiers formés en "cloche Étroite" et plantés à fortes densités nous ont avoué que c'est à leur $6 e^{\prime}$ feuillaison que leurs pommiers ont atteint leur seuil de rentabilité. Quelques-uns entre autres, selon nos estimations de récoltes, ont pu recueillir lors de la cinquième ou de la sixième feuillaison de leurs pommiers nains entre 30000 et $45000 \mathrm{~kg} / \mathrm{ha}$ chez les cultivars 'Spartan', 'McIntosh', 'Lobo' et 'Délicieuse rouge' formés en "cloche étroite" et plantés à des taux de 2000 à 3000 pommiers à l'hectare. A ce compte, s'il est vrai que le pomiculteur québécois en 1980 doit compter sur 20000 boisseaux de pommes pour obtenir un revenu convenable, il ne lui faudrait que 20 acres ( 8,1 hectares) de pommiers formés en "cloche étroite" et plantés à forte densité pour obtenir un tel revenu vers la $6^{\mathrm{e}}$ ou $7^{\mathrm{e}}$ feuillaison.

En ce qui concerne la production québécoise des poires, des, prunes et des cerises, notre volume de production a été si négligeable,du moins au cours des dernières décennies, que les relevés statistiques ne font même pas mention de ces cultures. Néanmoins, en 1976, la consommation per capita au Québec a été 7,2 livres de poires, 2,2 livres de prunes et 1,9 livres de cerises (douces et amères), en comparaison de 41 livres de pommes. Notre marché pourrait donc absorber un bon volume de production locale de poires, prunes et cerises en autant que la qualité de ces fruits soit adéquate.

Sous nos conditions climatiques, les cultivars de poires qui au point de vue rendement et qualité pourraient fournir des fruits concurrentiels sur les marchés locaux (P1. III, figs b, c, et d) sont le 'Favorit de Clapp', le 'Beauté Flamande', le 'Beurrée d'Anjou' et le 'Miney'. Bien que le 'Bartlett' soit un cultivar plus connu des gens d'ici, sa culture 
demeure beaucoup plus aléatoire car l'arbre est sensible au gel et à la brûlure bactérienne.

En ce qui concerne les prunes, les cultivars rustiques les plus susceptibles de trouver preneur sur le marché local sont 'Mont Royal', 'Reine Claude de Montmorency' et 'Damas Bleue'*. Beaucoup d'autres cultivars pourraient devenir d'acceptation commerciale puisqu'ils ont donné, au Québec, de bons rendements et des fruits de qualité (PI. III, figs e, f et $\mathrm{g}$ ). Un bulletin sur la culture de la prune au Québec est en préparation à la Station de Recherches de Saint-Jean; on y trouvera de plus amples informations sur le sujet.

Nous en sommes encore loin de 1a production commerciale des griottes et des cerises douces car ces deux cultures, surtout celle des cerises douces, sont très marginales. Cependant, il y aurait lieu d'intensifier la culture des griottes dans le Bas-Saint-Laurent et même ailleurs où elle existe déjà à 1 'état embryonnaire et où les cultivars 'Montmorency', 'Meteor' et 'North Star' se sont avérés bien adaptés (P1. III, fig a). Comme la compétition est très forte dans le domaine de la production des griottes en Amérique du Nord, une étude sérieuse des possibilités de mise en marché de ce fruit au Ouébec s'impose avant de préconiser l'établissement de plantations commerciales.

* noter que 'Damas Bleue' est une sélection de 'Damson' et est de qualité supérieure à cette dernière 


\section{Annexe}

Question et réponses concermant les diapositives montrées au pavizzon l'Amare le 21 août 1980

Q. Parmi les cultivars de pommiers employés au Québec, quels types de fructification pouvons-nous rencontrer?

R. Les types de fructification que l'on y trouve sont:
a) Type I
Les types à lambourdes (P1. I, figs a et b)
b) Type II
Les types McIntosh et Spartan (P1. I, figs c et d)
c) Type III
Le type Empire et Golden Delicious (P1. I, fig.e)
d) Type IV
Le type Cortland et Red Haralson (P1. I, figs.f et g)

Ces types correspondent quelque peu à ceux décrits par monsieur Lespinasse. En raison de la disposition des coursonnes dans la ramure de chaque type d'arbre, il faudra utiliser en verger quatre modes de conduite (taille et formation) différents.

Q. Au Québec, est-il plus important de drainer les sols à verger lorsqu'il s'agit de pommiers nains que lorsqu'il s'agit de pommiers de taille conventionnelle?

R. En raison des fortes précipitations en neige et en pluie, il est tout à fait primordial de bien drainer nos sols à verger au Québec et plus particulièrement s'il s'agit de pommiers nains car chez ceux-ci l'action néfaste des gels et dégels successifs des racines de l'arbre est beaucoup plus à craindre lorsqu'un sol est gorgé d'eau. Puisque les pommiers nains ont un système radiculaire peu profond et peu développé ils seront, en maintes occasions, plus sujets à l'asphyxie des racines due à un excès d'eau de surface (Pl. II, fig.a).

Q. Est-il nécessaire d'irriguer les pommiers nains?

R. Les pommeraies du Québec reçoivent chaque année une abondante précipitation mais malheureusement cette précipitation n'est pas 
uniformément distribuée. Il faut donc, dans la grande majorité des cas, avoir recours à l'irrigation, du moins lorsque la plantation en est à ses premières années; les jeunes pommiers ont un système radiculaire peu développé et inapte à bien explorer les réserves d'eau du sol qui, par temps de sécheresse, se rencontrent dans une zone plus ou moins profonde. Il est important de noter que l'équilibre physiologique d'un pommier nain est beaucoup plus facile à maintenir lorsqu'on utilise l'irrigation et, de ce fait, les arbres rapportent plus tôt et plus abondamment. D'ailleurs, certains cultivars nouveaux comme le Spartan, 1''Empire' et le 'Red Haralson' nécessitent 1'utilisation d'un système d'irrigation sans quoi, dans bien des cas, le fruit est de faible calibre à la récolte (P1. II, fig.b).

Q. Doit-on renoncer à planter des pommiers nains parce que, sous nos conditions, les branches des jeunes arbres sont très souvent brisées ou cassées par la glace, la neige et le verglas?

R. Certes le bris des branches par la glace, la neige et le verglas sont un handicap au Québec mais, en général, ces dommages en sont pas très considérables. D'ailleurs, même les pommiers standards, lorsqu'ils sont jeunes, peuvent être endommagés par la glace, la neige et le verglas. Pour contrer cette difficulté on fera bien d'employer de bonstuteurs ou un système de palissage solide. Les arbres peu ou pas supportés sont beaucoup plus affectés par ces dommages mécaniques. Le pomiculteur vigilant devra, dès qu'il se produit un dégel au cours de 1 'hiver libérer les branches des pommiers recouvertes de la neige molle. Après le durcissement de cette neige il deviendra impossible d'en retirer les rameaux. D'ailleurs, lorsque la neige fondante gèle elle écarte les branches du tronc avec une très grande force. Un autre phénomène peut aussi se produire au printemps lors de la fonte de la neige, soit la formation d'un vide sous la couche de glace retenant prisonnière les branches de pommier. C'est alors que le poids de la glace, qui fond moins vite que la neige, va entrainer et casser ces branches (P1. II, fig.e). 
Q. Est-ce que les dommages causés par les campagnols des champs et le cerf de Virginie (chevreuil) sont plus à craindre chez le pommier nain?

R. Etant donné la forte densité et la petite taille des pomniers des nouvelles plantations, ils sont certainement beaucoup plus vulnérables aux assauts de ces deux ravageurs. Il importe donc de bien protéger les pommiers à cet effet.

Q. Les pommiers nains sont-ils plus sensibles à la brûlure bactérienne que les pommiers de taille régulière?

R. La brûlure bactérienne n'envahira pas plus le pommier nain que le pommier standard pour autant que la fertilisation azotée n'est pas trop élevée, qu'on utilise pas des cultivars très sensibles (Jaune Transparente, Saint-Laurent, etc.) et qu'on élimine les sources de contamination. La brûlure bactérienne peut se propager par la taille d'été à partir d'un foyer d'infection, taille qui se pratique aussi bien sur les pommiers nains que sur les pommiers standards.

Q. La culture du prunier au Québec est-elle possible malgré les maladies qui menacent cette culture?

R. Malgré la présence du nodule noir (Dibotryon morbosum), de la tumeur du collet (Agrobacterium tumefaciens) et des viroses, la culture du prunier est possible au Québec. On peut maintenant se prémunir contre ces maladies. Un bulletin, en préparation à la Station de Recherches de Saint-Jean, traitera de la culture des pruniers au Québec, ainsi que des méthodes de lutte contre les maladies.

Q. Que faire pour prévenir l'affranchissement du scion sur porte-greffe nanisant?

R. L'affranchissement, c'est-à-dire la formation de racines issues du scion peut être évité lors de la plantation du pommier en plaçant la 
zone de souture de la greffe à au moins 10 centimètres au-dessus de la surface du sol (P1. II, fig.c). Si par erreur la souture se trouvait dans le sol et que des racines avaient commencé à se développer, il faudrait couper ces racines au plus tôt et dégager la zone de la greffe de tout le sol qui la recouvre. Si on ne les enlève pas, les racines d'affranchissement, après un an ou deux, auront déjà fait mourir complètement le porte-greffe nanisant (Pl. II, fig.c).

Q. Le désherbage est-il important au pied d'un pommier nain?

R. Dans des parcelles expérimentales à Frelighsburg, nous avons noté que là où on a laissé croître les mauvaises herbes près du tronc des jeunes pommiers, ceux-ci se sont très peu développés et n'ont même pas commencé à rapporter à leur quatrième feuillaison. Par contre, dans les mêmes parcelles expérimentales où nous avons détruit chimiquement les mauvaises herbes de façon à ce que le sol soit nu totalement, c'est-à-dire pendant toute la saison de végétation ou partiellement, c'est-à-dire seulement jusqu'à la mi-août, le développement des arbres dans 1 'un et 1 'autre cas a été de 2 à 3 fois supérieur à celui des pommiers non désherbés et la récolte a varié entre 5 et 12 kilogrammes de fruits par pommier chez les cultivars 'McIntosh', 'Jerseymac' et 'Red Delicious'.

Q. Y a-t-il un moyen d'éliminer la formation de galles radiculaire aériennes burr knots chez certains porte-greffes de pommier?

R. Il s'est fait relativement peu de recherches dans ce domaine. Toutefois le docteur C.G. Forshey et monsieur F. McNicholas, dans l'est de l'état de New York, ont trouvé que des applications d'acide naphtalène acétique (ANA ou NAA) à 1 \% ont empêché les galles radiculaires aériennes de se développer chez le porte-greffe 'M 26 ' (Pl. II, fig.d). Il a été précédemment question de ce traitement (voir sous le 'Malling $26^{\prime}$ ). 
Q. L'insolation qui se produit à la fin de $I^{\prime}$ hiver est-elle à craindre chez les jeunes pommiers nains au Québec?

R. Il arrive souvent que l'insolation de la fin de l'hiver ou 'brûlure du sud-ouest' soit un très sérieux problème chez les jeunes pommiers au Québec. Pour obvier à cette difficulté, nous conseillons de placer les tuteurs du côté sud-ouest de 1'arbre lors de la plantation, ou encore de badigeonner le tronc du pommier avec un mélange de peinture à prix modique d'intérieur ou d'extérieur de type latex diulée (2 pintes d'eau par gallon) à laquelle on ajoute 2 livres de Thiram (75\% W.P.) sous forme de poudre mouillable. Le Thiram agit comme répulsif au mulot (campagnol des champs).

N.B. Il est important d'éviter l'utilisation de peinture à prix élevé au latex à base d'alkyle (mildioucide)。 L'alkyle est toxique aux arbres fruitiers.

Q. Quelles sont les carences les plus fréquentes affectant les jeunes pommiers nains au Québec?

R. Au Québec, les carences en magnésium et en bore sont très fréquentes (P1. II, figs h, i). Il arrive parfois que le calcium soit déficitaire. On consultera le Guide de la culture du pommier au Québec à ce sujet (Publication 333 du ministère de 1'Agriculture du Québec Agdex $211 / 20)$.

Q. Doit-on utiliser un brise-vent pour protéger les nouvelles plantations de pommiers nains au Québec?

R. L'emploi d'un brise-vent est primordial dans les pommeraies du Québec. On doit placer le brise-vent du côté ouest du verger d'est-à-dire du côté du vent dominant. Cependant, il est à conseiller de ne pas planter de pommier trop près du brise-vent sans quoi les accumulations de neige au cours de nos hivers auront tôt fait de casser les branches des pommiers. Un bon brise-vent pourrait se composer d'une rangée d'arbres à feuilles caduques et d'une rangée de conifères plantés à $I^{\prime}$ arrière plan et en quinconce. 
Références

1 ANON. 1978. Coup d'oeil sur 1'agro-alimentaire. Agriculture Québec. Service des études économiques. 334 p., Fruits p. 258.

2 BLAIR, D.S. 1954. Plums for cold areas of Eastern Canada. Can. Dep. Agr. Pub. 849,14 p.

3 DAVIS, M.B.; D.S. Blair and H.B. Cannon. 1934-1948. Canada Dept. Agr. Div. Hort. Progr. Rept. pp. 5-14.

4 FISHER, D.V. 1969. Capitalization at the break-even point for a high density and low density apple orchard. Report of the C.D.A. Res. Stn., Summerland, B.C.

5 GARON, J. et F. Ouellet. 1978. Réorientation de I'Industrie Pomicole au Québec. Programme Agricole 03, Elément 02, Service des productions végétales. Agriculture Québec, 2 p.

6 GRANGER, R.L. 1972. Hints on the use of apple rootstocks. Meeting on Apple Orchard Management (held in Ottawa on November).

7 GRANGER, R.L. 1977. Problems and progress with high density apple orchards in Québec, p. 21 in Hort. Res. Inst. of Ontario, Vineland Station, Report on workshop on Apple Management, August 17-19, $78 \mathrm{p}$.

8 GRANGER, R.L.; G. Rousselle et M. Lareau. 1974. L'Ottawa 3, le Malling 9 et le Malling 26 en tant que porte-grefies et tiges intermédiaires de pommier. Québec Horticole, pp. 12-16.

9 HUTCHINSON, A. 1964. Rootstocks for tree fruits. Hort. Exp. Stn., Vineland Ont. Dep. of Agr. Fub. 334, 18 p.

10 HUTCHINSON, A. and E. Andersen. 1976. Compact apple orchard experiments, management, costs and returns the first 5 years. Comm. given at the International Dwarf Fruit Tree Association, Michigan.

11 LAPINS, K. 1963. Cold hardiness of rootstocks and framebuilders for tree fruits. Res. Stn. Summerland.

12 LORD, W.J. et J. Costante. 1977. Establishment and management of compact apple trees. Coop. Ext. Service, Un. of Mass. U.S.P.A. and County Ext. Serv. Coop. 32 p.

13 MARGOLIN, A.F. 1951. Dwarf apples in the Moscow region (in Russian) Sad i Ogorod 12: 5-7 1950. Plant Breeding abstracts 21: 1364. 
14 MILLER, S.R. 1976. Economics involved in apple bed plantings. Report of the res. stn of Smithfield, Ontario, $14 \mathrm{p}$.

15 NORTON, R.L. 1971. What you must know about high density plantings. Go from red to black. July issue of the Am. Fruit Grower, p. 10-14.

16 ROUSSELLE, G.L.; R.L. Granger; C.N. Fortin et S. Riendeau. 1975-76. Résumé des recherches en cultures fruitières - Station de Recherches de Saint-Jean. Vols 4 et 5, p. 33 .

17 SMIRNOV, V.F. 1960. Cultivation of dwarf fruit trees (in Russian). Gos. Izd. Selskokhoz. Liter., Moskwa, 4th ed.

18 TAPER, C.D. 1979. Personal communication. Re: progress report on the performance of dwarf and semi-dwarf apple trees at Macdonald College.

19 TUKEY, H.B. 1964. Dwarfed Fruit Trees. Mich. State University. The Macmillan Co. New York. Textbook, $562 \mathrm{p}$.

20 TUKEY, R.L. 1971. Proc. B.C. Fruit Growers Association. Hort. Forum, p. 35.

21 WATKINS, R. 1968. Summary of results on some of the most promising Ottawa clonal apple rootstocks with Malling and Malling Merton rootstocks for comparison, Report of Jan. 11. 



\section{Planche 1}

Types de fructification et formes de pommiers au Québec.

a. 'MorSpur McIntosh'/'M. robusta $5^{\prime}$ à sa $8^{\text {e }}$ feuillaison

b. Rameaux de 'Mac Spur McIntosh'

c. 'Imperial Allred McIntosh'/'Ott $3^{\prime}$ à sa $4^{\mathrm{e}}$ feuillaison

d. 'Spartan'/'M 26' à sa $4^{\mathrm{e}}$ feuillaison

e. 'Empire'/'Ott $8^{\prime}$ à sa $8^{\mathrm{e}}$ feuillaison

f. 'Red Haralson'/'Ott 8' à sa 8 e feuillaison

g. 'Cortland'/'M 9 ' à sa $22^{\mathrm{e}}$ feuillaison

h. 'Imperial Allred McIntosh'/'Ott 3' formé en "cloche étroite" à sa $4^{\mathrm{e}}$ feuillaison

i. 'Golden Delicious'/'M 9' formé en "palmette de Van Roechoudt" à sa 6e feuillaison

j. 'Spartan'/'Ott 3' formé en "palmette oblique" à sa $4^{e}$ feuillaison k. 'Imperial Allred McIntosh'/'Ott 3' formé en arbre de "plein vent" 

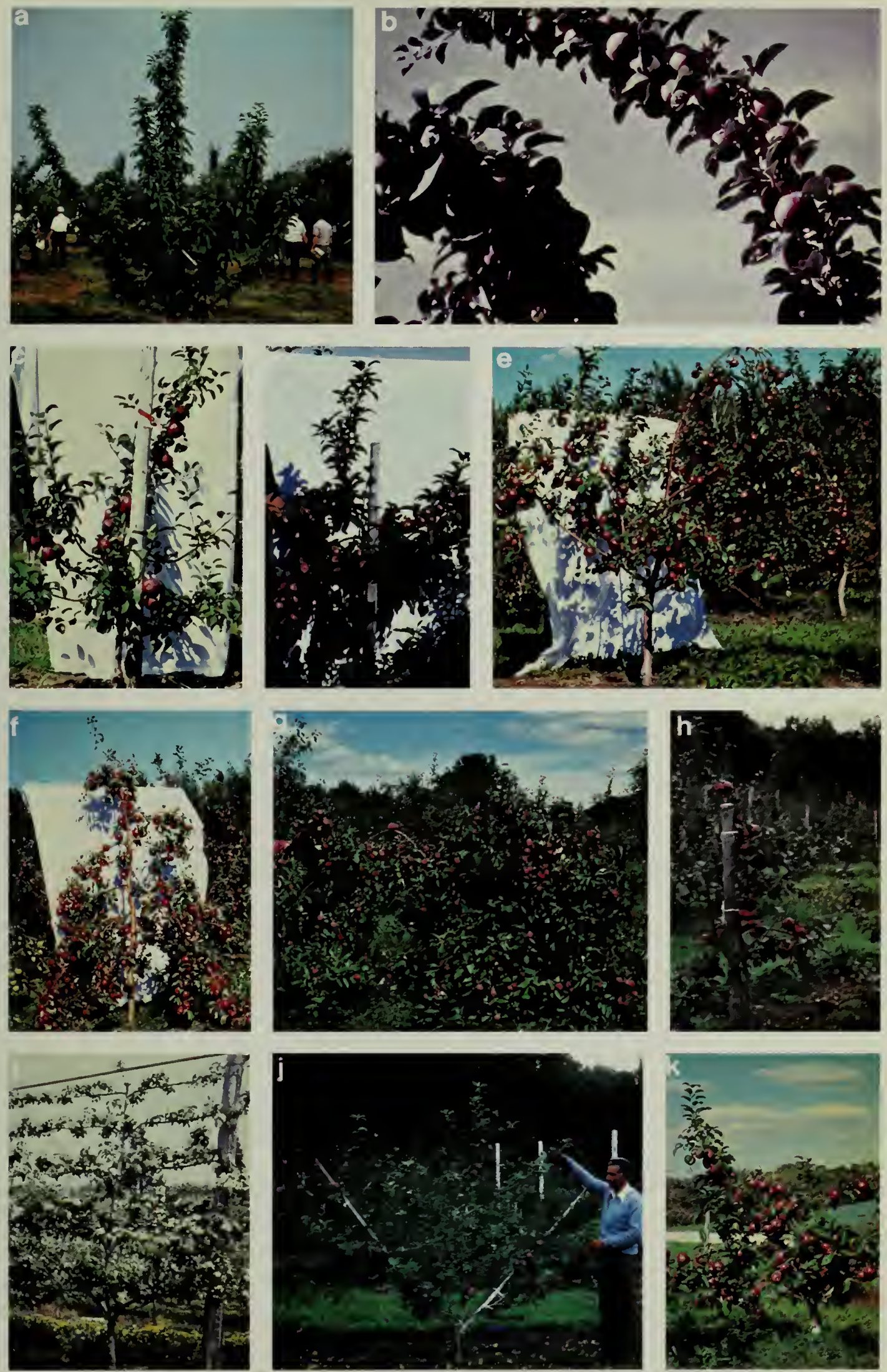


Certaines anomalies ou particularités des vergers à fortes densités au Québec.

a. Mauvais égout de surface lors du mois de juin

b. Goutteur d'un système d'irrigation goutte à goutte

c. Affranchissement d'un pomier 'Imperial Allred McIntosh'/'M 26'

d. Galles radiculaires aériennes sur 'M 26 '

e. Bris de branches d'un 'McIntosh'/'M $9^{\prime}$ après sa $15^{\mathrm{e}}$ feuillaison

f. Pommier 'Spartan'/'M 9 ' de plein vent

g. Flèche ployant sous le poids des pommes car le tuteur trop court du pommier ne peut pas l'assujettir

h. Carence en magnésium

i. Carence en bore 


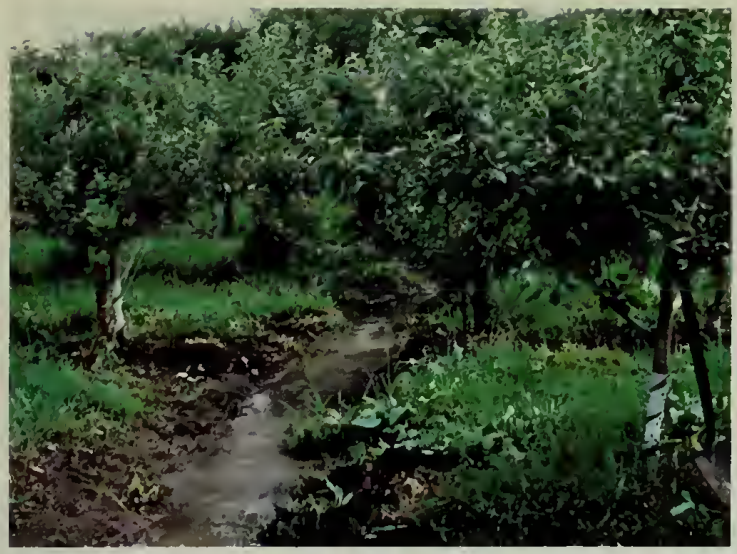

$10 x=-17$ z

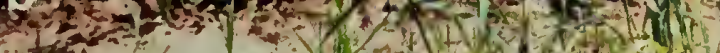

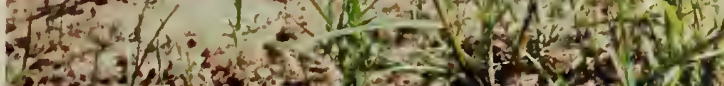

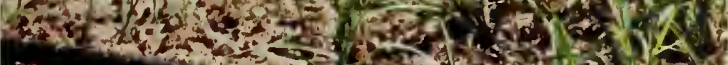

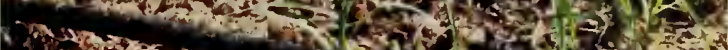

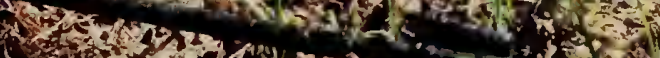

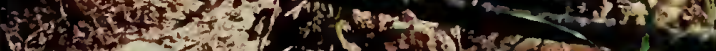

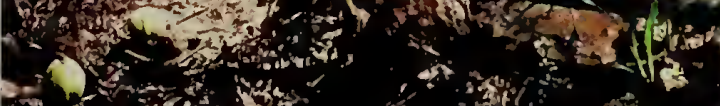

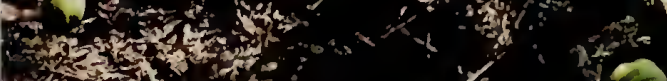

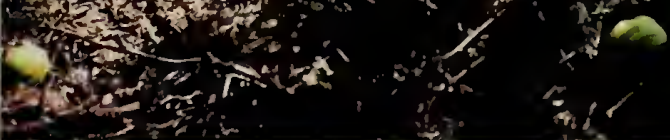
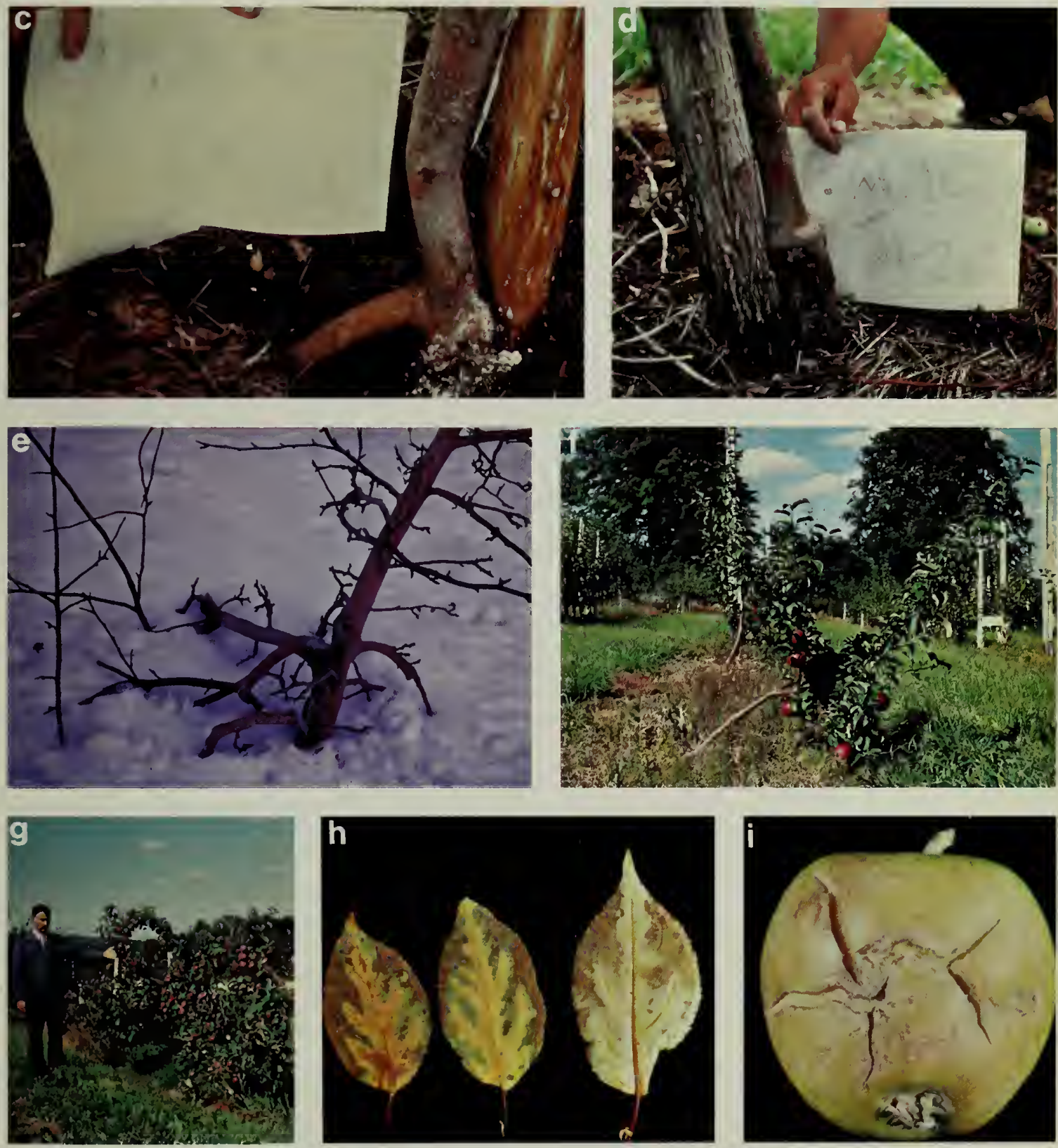

Cerises, poires, prunes et tumeur du collet.
a. Griottes 'Montmorency'
b. Poires 'Favorites de Clapp'
c. Poirier du cultivar 'Favorite de Clapp'
d. Poires 'Miney'
e. Prunes 'Early Italian'
f. Prune 'Kahinta'
g. Prunier 'Kahinta' à sa $8^{e}$ feuillaison
h. Tumeur du collet sur racines de pruniers 'Brompton' 

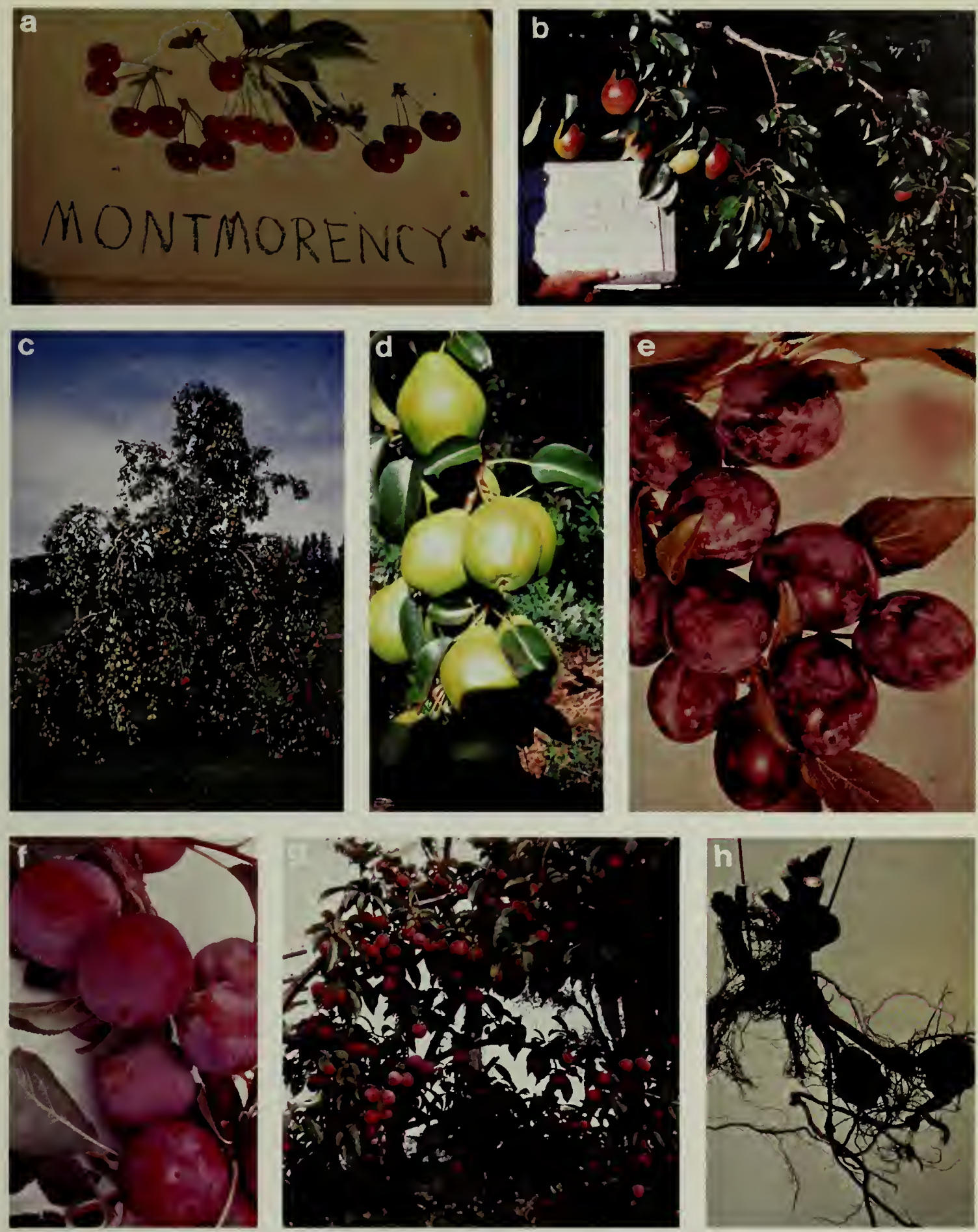


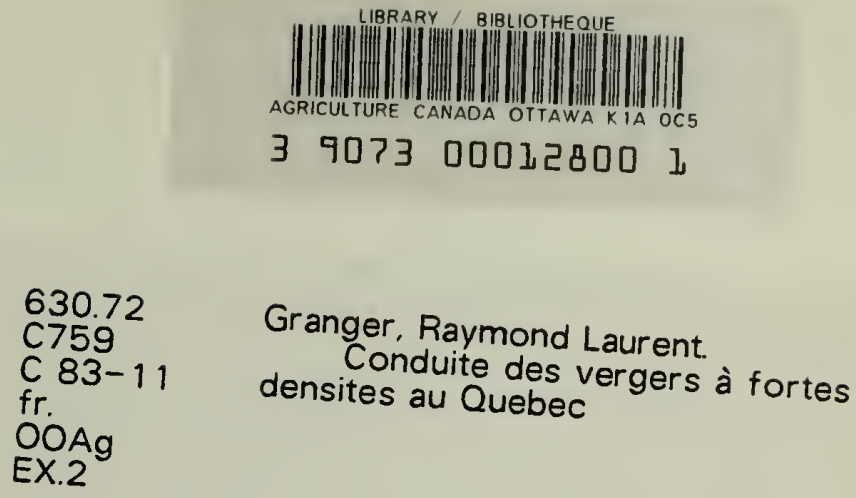
